\title{
Concentração e retenção do selênio em peixes marinhos
}

\author{
Concentration and retention of selenium in marine fish
}

\section{Alfredo TENUTA FILHO ${ }^{1 *}$, Luciene Fagundes Lauer MACEDO $^{1}$, Déborah Inês Teixeira FAVARO ${ }^{2}$}

\section{Resumo}

O pescado contém substancialmente maiores concentrações de selênio (Se) do que outras carnes e é, por isso, considerado a maior fonte dietária desse nutriente. Ao mesmo tempo, o pescado também é a maior fonte de ingestão de mercúrio (Hg) na dieta humana, cuja toxidez pode ser reduzida pelo Se, por interação entre ambos. Em consequência, a biodisponibilidade do Se é reduzida. No presente estudo, a ocorrência do Se foi avaliada em espécies marinhas de peixes brasileiros, comercialmente disponíveis, e determinada sua retenção em cação-mangona. Os níveis médios de Se encontrados nos peixes ósseos, corvina (Micropogonias furnieri), peixe-porco (Balistes capricus), pescada-branca e sardinha (Sardinella brasiliensis), 0,48 $\pm 0,18 \mu \mathrm{g} \cdot \mathrm{g}^{-1}$, e nos peixes cartilaginosos, cação-anjo, cação-cambeva, cação-machote e cação-mangona, $0,36 \pm 0,07 \mu \mathrm{g} \cdot \mathrm{g}^{-1}$, não diferiram entre si $(\mathrm{p}>0,05)$ e situaram-se na mesma faixa de ocorrência indicada na literatura para peixes marinhos em geral. Os níveis de Se detectados nos cações (espécies predadoras) não permitem o estabelecimento de relação estequiométrica suficiente para a interação com o Hg, visando à proteção tóxica esperada. A retenção efetiva do Se medida em cação-mangona, após extração com água, foi considerada elevada (mínimo de $68,81 \%$ ) e importante sob os pontos de vista nutricional e toxicológico.

Palavras-chave: selênio; peixe marinho; mercúrio.

\begin{abstract}
Fish contains considerably higher selenium (Se) concentrations than other kinds of meats, and thus it is considered a major dietary source of this nutrient. At the same time, fish is also the major source of mercury $(\mathrm{Hg})$ in the human diet, whose toxicity can be reduced by the Se through the interaction between both compounds. In consequence, the biological availability of Se is reduced. In the current study, the occurrence of Se was evaluated in commercially available Brazilian marine species, and the retention of Se in sharks (sand tiger shark) was determined. The average levels of Se found in bony fish, gutted corvina (Micropogonias furnieri), triggerfish (Balistes capricus), hake and sardine (Sardinella brasiliensis), $0.48 \pm 0.18 \mu \mathrm{g} . \mathrm{g}^{-1}$, and in cartilaginous fish, small shark (squantina Guggenheim), hammerhead shark, spinner shark, and sand tiger shark, $0.36 \pm 0.07 \mu \mathrm{g} \cdot \mathrm{g}^{-1}$, did not differ from one another $(\mathrm{p}>0.05)$. In fact, their occurrence was in accordance with the literature data on marine fish. The levels of Se found in the sharks (predatory species) are not enough to establish a stoichiometric relation for the interaction with $\mathrm{Hg}$ focusing on the desired toxic protection. The effective Se retention evaluated in the sand tiger shark, after extraction with water, was considered high (minimum of 68.81\%) and important taking nutritional and toxicological aspects into account. Keywords: selenium; marine fish; mercury.
\end{abstract}

\section{Introdução}

O selênio ( $\mathrm{Se}$ ) é um micronutriente essencial, está incorporado ao sítio ativo de selenoproteínas, entre as quais importantes enzimas antioxidantes, reduzindo riscos de aterosclerose e câncer. Atua também no catabolismo hormonal, sistema imunológico, fertilidade, reprodução e metabolismo muscular (BROWN; ARTHUR, 2001; RAYMAN, 2000) e, se ocorrer em concentrações elevadas, é reconhecido como um agente tóxico (LALL, 1995).

O pescado, por conter substancialmente maiores concentrações de Se do que outros alimentos cárneos, é considerado a maior fonte dietária desse nutriente (LALL, 1995). Ao mesmo tempo o pescado é a maior fonte de mercúrio $(\mathrm{Hg})$ na dieta humana, em consequência de sua vulnerabilidade natural, ocorrida por contaminação cumulativa através da cadeia trófica
(TENUTA-FILHO; NASCIMENTO, 2007). Paralelamente, tem sido considerado que o $\mathrm{Hg}$ ou o metilmercúrio $(\mathrm{MeHg})$ combinados com o Se, em alimentos de origem marinha, protegem o consumidor contra a ação tóxica do Hg. Esta complexação incidentalmente reduz a biodisponibilidade do Se (RAYMAN, 2000). Por sua vez, nenhuma propriedade nutricional ou biológica benéfica é atribuída ao $\mathrm{Hg}$.

Como na grande maioria do pescado marinho o Se muscular está estequiometricamente em excesso em relação ao $\mathrm{Hg}$, tem sido considerado que o consumo de peixes, crustáceos e moluscos não expõe o consumidor aos efeitos tóxicos do Hg. A interação entre $\mathrm{Hg}$ e Se tem sido estudada, mas não o suficiente para o necessário entendimento da ação protetora do Se (PEAKALL; BURGER, 2003; RUITER, 1995).

Departamento de Alimentos e Nutrição Experimental, Faculdade de Ciências Farmacêuticas, Universidade de São Paulo - USP, Av. Prof. Lineu Prestes, 508, Bloco 14, CEP 05508-900, São Paulo - SP, Brasil,E-mail: eetenuta@usp.br

${ }_{2}^{2}$ Comissão Nacional de Energia Nuclear, Instituto de Pesquisas Energéticas e Nucleares, Av. Prof. Lineu Prestes, 2242, CEP 05508-900, São Paulo - SP, Brasil

${ }^{*}$ A quem a correspondência deve ser enviada 
No entanto, particular preocupação deve ser reservada aos peixes predadores - entre os quais, principalmente, o cação -, que têm a capacidade de acumular Hg muscular em excessivas e perigosas quantidades, condição esta que os torna impróprios para serem usados como alimento (TENUTA-FILHO; NASCIMENTO, 2007).

Informações insuficientes quanto à ocorrência do Se em pescado originário de águas brasileiras motivaram este estudo, empregando espécies marinhas de peixes disponíveis comercialmente, tendo sido avaliada também a sua retenção em cação-mangona.

\section{Material e métodos}

\subsection{Amostras}

Aleatoriamente, foram adquiridas amostras congeladas em mercados e feiras livres do bairro de Pinheiros, em São Paulo - SP, Brasil, nos períodos de março a novembro de 2005 e 2006: corvina (Micropogonias furnieri) $(\mathrm{n}=5)$, peixe-porco (Balistes capricus) $(\mathrm{n}=5)$, pescada-branca $(\mathrm{n}=5)$ e sardinha (Sardinella brasiliensis) $(\mathrm{n}=5)$ - peixes ósseos -; e, cação-anjo $(\mathrm{n}=5)$, cação-cambeva $(\mathrm{n}=10)$, cação-machote $(\mathrm{n}=8)$ e cação-mangona $(\mathrm{n}=12)$ - peixes cartilaginosos. Cada amostra analisada correspondeu aos filés ou postas, triturados e homogeneizados, derivados de seis espécimes de cada espécie óssea ou cartilaginosa, respectivamente. Por dificuldade técnica, nem todas as espécies puderam ser identificadas zoologicamente. No caso dos cações, deveu-se ao fato de serem comercializados já descabeçados, eviscerados e sem barbatanas ventrais, dorsais e caudal em operações realizadas a bordo das embarcações pesqueiras, impedindo assim a identificação zoológica da espécie.

\subsection{Composição química}

A umidade, as cinzas e os lípides totais foram quantificados por gravimetria, após a eliminação dos voláteis a $105{ }^{\circ} \mathrm{C}$, incineração a $560{ }^{\circ} \mathrm{C}$ (AOAC, 1995) e extração em clorofórmio/metanol (FOLCH; LESS; STANLEY, 1957), respectivamente, e a proteína determinada em relação ao nitrogênio total, após digestão ácida e destilação em meio alcalino, por arraste a vapor, empregando o fator de conversão de 6,25 (AOAC, 1995).

\subsection{Selênio}

A amostra liofilizada $(0,5-1 \mathrm{~g})$ foi digerida com mistura (20 mL) dos ácidos nítrico (65\%, Merck, 100456), perclórico (70-72\%, Merck, 100519) e sulfúrico (95-97\%, Baker, 9673-33), na proporção de 20:5:2 (v:v:v), respectivamente, até o início da volatilização do ácido sulfúrico $\left(\sim 290{ }^{\circ} \mathrm{C}\right)$. O Se VI foi reduzido a Se IV e colocado para reagir com o 2,3-diamino1,4-dibromonaftaleno ( $\mathrm{Br}_{2}$-DAN), formando o 4,7-dibromo5,6-benzopiazselenol ( $\mathrm{Br}_{2}$-DAN-Se), cuja fluorescência serviu de medida do Se total, em espectrofluorímetro Hitachi modelo F-3010 (JOHANSSON; LUO; OLIN, 1995). O Br - -DAN foi sintetizado a partir do 2,3-diaminonaftaleno (DAN) (Sigma, D-2757) (ZHENG et al., 1986).

\subsection{Retenção do selênio}

A retenção (aparente e efetiva) do Se foi avaliada em músculo de cação-mangona previamente triturado em moedor de carne comum, empregando-se um crivo de $1 \mathrm{~cm}$ de diâmetro. O músculo triturado foi então extraído com água destilada, numa relação $1: 10(\mathrm{~m} / \mathrm{v})$, durante 30 minutos, e sob agitação eletromagnética suficiente para permitir movimentação adequada da amostra. Ao final, o resíduo sólido resultante foi recuperado por centrifugação $(12.000 \times \mathrm{g} / 10$ minutos $)$. A retenção efetiva levou em conta a perda de sólidos, por solubilização de minerais e proteínas (sarcoplasmáticas) e arraste de lípides, de acordo com Murphy, Criner e Gray (1975). A perda de sólidos considerada foi de $28,46 \mathrm{~g} .100 \mathrm{~g}^{-1}$, em matéria seca, sendo $6,10 \mathrm{~g}$ em minerais, $19,41 \mathrm{~g}$ em proteínas (correspondente a $25 \%$ da proteína total, segundo HAARD, 1995) e 2,95 g em lípides.

A retenção do Se foi avaliada também após a remoção do $\mathrm{Hg}$, empregando-se a amostra anteriormente triturada e livre de tecido conjuntivo (obtida por peneiramento em tela de náilon, com diâmetro de $1 \mathrm{~cm}$ ). A amostra foi então extraída com boro-hidreto de sódio a 3\%, na proporção de 1:10 (m/v), por 20 minutos, seguido do ajustamento do $\mathrm{pH}$ a 4,5, com ácido clorídrico $6 \mathrm{~N}$, com base em Cohen e Schrier (1975). Em seguida, a amostra foi centrifugada (12.000 xg/10 minutos), ressuspensa em água destilada e novamente centrifugada. As operações de ressuspensão em água destilada e a centrifugação correspondente foram repetidas uma vez mais. O conteúdo do Hg na amostra e no produto obtido foi quantificado por espectrometria de absorção atômica com geração de vapor frio (CV AAS), usando-se equipamento da Perkin Elmer, modelo 3110, acoplado a um sistema de injeção de fluxo FIAS 200, conforme Kitahara et al. (2002). Na avaliação da retenção efetiva do Se, após a remoção do $\mathrm{Hg}$, foi levada em conta somente a perda de minerais e lípides, de $4,11 \mathrm{~g} .100 \mathrm{~g}^{-1}$, em matéria seca, já que o processo usado permitiu a recuperação das proteínas a $\mathrm{pH} 4,5$.

\section{Resultados e discussão}

\subsection{Concentração do selênio}

A ocorrência do Se foi de 0,34 a $0,64 \mu \mathrm{g} \cdot \mathrm{g}^{-1}\left(0,48 \pm 0,18 \mu \mathrm{g} \cdot \mathrm{g}^{-1}\right)$ nos peixes ósseos (corvina, peixe-porco, pescada-branca e sardinha) e, de 0,29 a $0,45 \mu \mathrm{g} \cdot \mathrm{g}^{-1}\left(0,36 \pm 0,07 \mu \mathrm{g} \cdot \mathrm{g}^{-1}\right)$ nos cartilaginosos (cação-anjo, cação-cambeva, cação-machote e cação-mangona), em matéria úmida, não tendo sido verificada diferença significativa $(p>0,05)$ entre os dois grupos considerados (Tabela 1). As espécies estudadas eram de significativa comercialização no município de São Paulo, em especial a sardinha, cuja produção nacional em 2007 foi prevista para 60 mil toneladas (FUNDACENTRO, 2007), uma das maiores nas duas últimas décadas.

Foi observada uma baixa variabilidade nos teores de Se encontrados na sardinha ( $\mathrm{CV}=7,81 \%)$, ao contrário da registrada em relação ao cação-anjo $(\mathrm{CV}=41,03 \%)$, caçãocambeva $(\mathrm{CV}=15,56 \%)$, cação-machote $(\mathrm{CV}=32,26 \%)$ e cação-mangona $(\mathrm{CV}=24,14 \%)$, sugerindo que isso possa ser 
reflexo dos hábitos alimentares das espécies envolvidas. No caso, sendo planctófaga, a sardinha, e carnívoras (predadoras), as espécies de cação.

Tem sido observada uma carência muito grande quanto ao conhecimento da ocorrência de Se em peixes de origem brasileira, tanto marinhos quanto de água doce, o que impede que isso possa ser usado plenamente para os propósitos inerentes, nutricionais ou não, tendo em vista a importância desse nutriente. $\mathrm{O}$ valor médio de $0,36 \pm 0,07 \mu \mathrm{g} \cdot \mathrm{g}^{-1}$ (em matéria úmida) encontrado nos cações (Tabela 1) foi bem superior ao relatado por Chicourel et al. (1995) para o caçãoazul (Prionace glauca), também comercializado em São Paulo, de $0,41 \pm 0,16 \mu \mathrm{g} \cdot \mathrm{g}^{-1}$ ( $\mathrm{n}=14$; em matéria seca).

Ferreira et al. (2002) quantificaram o Se em vários alimentos consumidos no Brasil. Em cação (posta) foram encontradas concentrações de $0,113 \mu \mathrm{g} \cdot \mathrm{g}^{-1}$; em camarão vermelho, $0,25 \mu \mathrm{g} \cdot \mathrm{g}^{-1}$; em merluza (filé), $0,283 \mu \mathrm{g} \cdot \mathrm{g}^{-1}$; enquanto em atum "sólido" enlatado, 0,525 $\mu \mathrm{g} . \mathrm{g}^{-1}$; em sardinha enlatada em óleo, $0,46 \mu \mathrm{g} \cdot \mathrm{g}^{-1}\left(0,305-0,614 \mu \mathrm{g} \cdot \mathrm{g}^{-1}\right)$; e em sardinha enlatada em molho de tomate $0,809 \mu \mathrm{g} \cdot \mathrm{g}^{-1}$.

Segundo resultados encontrados em literatura, publicados por vários autores e reunidos por Cappon (1990), Lall (1995), Wheaton e Lawson (1985), e os obtidos por Plessi, Bertelli e Monzani (2001) e Vlieg (1990), para espécies de peixes marinhos foram encontrados níveis de Se entre 0,11 e 1,40 $\mu \mathrm{g} . \mathrm{g}^{-1}$, em matéria úmida. Em geral, os teores de Se em pescado são apreciavelmente altos sem, contudo, representarem risco tóxico ao consumidor, sendo que esta variabilidade é decorrente de fatores bioecológicos envolvendo a espécie, região geográfica e hábito alimentar, entre outros (FOSTER; SUMAR, 1997).

\subsection{Retenção do selênio}

A retenção do Se foi avaliada após a extração com água e remoção do $\mathrm{Hg}$ com $\mathrm{NaBH}_{4}$, verificada em cação-mangona (Tabela 2). A solubilidade do Se em pescado, se elevada, tem

Tabela 1. Selênio em peixes ósseos e cartilaginosos, comercializados em São Paulo.

\begin{tabular}{lcc}
\hline Peixes & $\begin{array}{c}\text { Se }\left(\mu \text { g.g }^{-1}, \text { matéria úmida }\right) \\
\text { Média } \pm \text { DP }(\mathrm{CV}, \%)\end{array}$ \\
\hline Ósseos & Corvina $(\mathrm{n}=5)^{(1)}$ & $0,62 \pm 0,08(12,90)$ \\
& Peixe-porco $(\mathrm{n}=5)^{(2)}$ & $0,34 \pm 0,06(17,65)$ \\
& Pescada-branca $(\mathrm{n}=5)^{(3)}$ & $0,30 \pm 0,05(17,67)$ \\
& Sardinha $(\mathrm{n}=5)^{(4)}$ & $0,64 \pm 0,05(7,81)$ \\
Cartilaginosos & Média $\pm \mathrm{DP}(\mathrm{CV}, \%)$ & $0,48 \pm 0,18(37,50)^{\text {a }}$ \\
& Cação-anjo $(\mathrm{n}=5)^{(5)}$ & $0,39 \pm 0,16(41,03)$ \\
& Cação-cambeva $(\mathrm{n}=10)^{(6)}$ & $0,45 \pm 0,07(15,56)$ \\
& Cação-machote $(\mathrm{n}=8)^{(7)}$ & $0,32 \pm 0,10(32,26)$ \\
& Cação-mangona $(\mathrm{n}=12)^{(8)}$ & $0,29 \pm 0,07(24,14)$ \\
& Média \pm DP $(\mathrm{CV}, \%)$ & $0,36 \pm 0,07(19,95)^{\text {a }}$ \\
\hline
\end{tabular}

$\mathrm{DP}=$ desvio padrão. $\mathrm{CV}=$ coeficiente de variação. $\mathrm{n}=$ número de repetições. Teores de umidade $={ }^{(1)} 79,30 \pm 1,08 ;^{(2)} 79,30 \pm 0,70 ;{ }^{(3)} 76,69 \pm 0,25 ;{ }^{(4)} 68,68 \pm 2,04 ;{ }^{(5)} 77,14 \pm 0,59$;

(6) $74,22 \pm 0,91$; ${ }^{(7)} 78,48 \pm 3,32 ;{ }^{(8)} 77,82 \pm 1,18$. Letras sobrescritas iguais na coluna indicam diferenças estatisticamente não significativas $(\mathrm{p}>0,05)$, avaliadas pela análise de variância (ANOVA), conforme o aplicativo $\operatorname{Excel}^{\circledR}$ (Microsoft). aspecto nutricional negativo pela possibilidade de perda durante o preparo para o consumo e, eventualmente, por ocasião da industrialização. Além disso, reduz a possibilidade de oferecer proteção em relação ao efeito tóxico provocado pelo Hg que contamina o pescado, em maior ou menor grau.

A retenção aparente do Se foi quase total, de $96 \%$, estando, no entanto, superestimada, por não ter sido levado em conta a perda de sólidos solubilizados (minerais e proteínas sarcoplasmáticas) e arrastados pela água (lípides), ocorrida durante a extração. A retenção aparente de micro e macronutrientes é maior que a real em alimentos cujos processamentos levam à perda de sólidos, implicando, necessariamente, que se conheça a perda de massa para então ser estabelecida a retenção efetiva (MURPHY; CRINER; GRAY, 1975). Desta forma foi constatada uma retenção efetiva de $68,81 \%$, considerada quantitativamente expressiva, podendo ser empregada como um valor mínimo em propósitos práticos, mesmo que subestimada. A subestimação indicada derivou principalmente de fatores não controlados como: (a) eram parciais a solubilidade de minerais e o arraste de lípides, mas foram calculados como totais; e, (b) as proteínas sarcoplasmáticas, formando a maioria dos sólidos perdidos pela solubilização em água, foram calculadas em $25 \%$, quando é sabido que estão presentes originalmente em músculo de pescado na ordem de $15-25 \%$ da proteína total (HAARD, 1995).

A retenção efetiva verificada pode ser explicada pelo fato de o Se estar incorporado a proteínas insolúveis (miofibrilares), em sua maioria. Os estados de oxidação do Se em compostos de ocorrência natural correspondem às formas $\mathrm{Se}^{2-}$ (seleneto, ex.: $\mathrm{Na}_{2} \mathrm{Se}$ ), Se elementar $\left(\mathrm{Se}^{0}\right), \mathrm{Se}^{4+}$ (selenito, ex.: $\mathrm{Na}_{2} \mathrm{SeO}_{3}$ ) e $\mathrm{Se}^{6+}$ (selenato, ex.: $\mathrm{Na}_{2} \mathrm{SeO}_{4}$ ), mas em alimentos normalmente só ocorrem como selenometionina e selenocisteína, incorporadas às proteínas, incluindo enzimas (REILLY, 1995).

Cappon (1990) relatou retenção de 36,5-51,3\% para o Se contido em peixes marinhos após a extração em água, ou seja, um valor bem menor que o do presente trabalho $(68,81 \%)$ (Tabela 2). Resultados conflitantes foram relatados na literatura, observados em pescado, obtidos quando empregados meios de extração envolvendo tampões, do que tudo indica por maior ou menor extratividade das proteínas presentes. Maher (1985) verificou que o Se estava predominantemente associado com

Tabela 2. Retenção do selênio em músculo de cação-mangona extraído com água e com boro-hidreto de sódio $\left(\mathrm{NaBH}_{4}\right)$.

\begin{tabular}{|c|c|c|c|c|}
\hline \multirow[t]{2}{*}{ Extração } & \multicolumn{2}{|c|}{$\begin{array}{l}\text { Se }\left(\mu g . g^{-1}, \text { matéria seca }\right) \\
\text { Média } \pm \text { DP }(C V, \%)\end{array}$} & \multicolumn{2}{|c|}{$\begin{array}{l}\text { Retenção do Se (\%) } \\
\text { Média } \pm \text { DP (CV, \%) }\end{array}$} \\
\hline & $\begin{array}{l}\text { Músculo } \\
\text { Controle }\end{array}$ & $\begin{array}{l}\text { Músculo } \\
\text { extraído }\end{array}$ & Aparente & Efetiva \\
\hline Água $(\mathrm{n}=10)$ & $\begin{array}{c}1,43 \pm 0,27 \\
(18,70)^{\mathrm{a}}\end{array}$ & $\begin{array}{c}1,38 \pm 0,26 \\
(18,68)^{\mathrm{a}}\end{array}$ & $\begin{array}{c}96,18 \pm 1,44 \\
(1,50)\end{array}$ & 68,81 \\
\hline $\mathrm{NaBH}_{4}(\mathrm{n}=5)$ & $\begin{array}{c}1,48 \pm 0,05 \\
(3,38)^{\mathrm{a}}\end{array}$ & $\begin{array}{c}0,93 \pm 0,03 \\
(3,23)^{\mathrm{b}}\end{array}$ & $\begin{array}{c}62,90 \pm 1,00 \\
(1,59)\end{array}$ & 60,31 \\
\hline
\end{tabular}

$\mathrm{DP}=$ Desvio padrão. $\mathrm{CV}=$ Coeficiente de variação. $\mathrm{n}=$ Número de repetições. Letras sobrescritas iguais, nas linhas, indicam diferença estatisticamente não significativa $(\mathrm{p}>0,05)$, ao contrário das diferentes $(\mathrm{p}<0,05)$, avaliadas pela análise de variância (ANOVA), conforme o aplicativo Excel ${ }^{\circ}$ (Microsoft). 
proteínas solúveis, extraídas com o tampão Tris/ $\mathrm{HCl} 0,1 \mathrm{M}, \mathrm{pH}$ 7,5 (contendo $0,1 \%$ de $\mathrm{NaCl}, 1 \%$ de dodecil sulfato de sódio e $0,05 \mathrm{M}$ de ditiotreitol). Assim, a solubilidade verificada foi de $72 \pm 4 \%$ em camarão (Penaeus latisulcatus), $80 \pm 5 \%$ em scallop (Pecten alba), $78 \pm 7 \%$ em lula (Sepioteuthis autralis) e $76 \pm 3 \%$ em peixe (Hemir hamphus australis). Åkesson e Srikumar (1994), empregando o tampão Tris/ $\mathrm{HCl} 10 \mathrm{mM}, \mathrm{pH}$ 7,4, opostamente ao que foi observado por Maher (1985), constataram a retenção de Se de 77,73 e 74\% em arenque (Clupea arengus) e de $81,85 \%$ e 78\% em bacalhau (Gadus morhua), frescos, refrigerados (por uma semana) ou congelados. Em plaice (Pleuronectes platessa) e em mackerel (Scomber scombrus) congelados a retenção de Se encontrada foi de 69 e 54\%, respectivamente, enquanto em plaice fresco foi de $40 \%$.

A extração no músculo do cação-mangona feita usando $\mathrm{NaBH}_{4}$ serviu para avaliar a retenção do Se e a remoção concomitante do $\mathrm{Hg}$, considerando-se o fato de ambos estarem complexados entre si, principalmente por conta da afinidade química apresentada pelo grupo sulfidrila $(-\mathrm{SH})$ da cisteína, na proteína muscular (TENUTA-FILHO; NASCIMENTO, 2007). A remoção do $\mathrm{Hg}$ com o $\mathrm{NaBH}_{4}$ é uma possibilidade de descontaminação proposta por Cohen e Schrier (1975), cujo resultado obtido no presente estudo foi de $\sim 86 \%$, avaliado em relação ao produto final. A retenção efetiva do Se, neste caso, resultou em $60,31 \%$, praticamente o mesmo valor da retenção aparente obtida, de $62,90 \%$ (Tabela 2).

Como no caso do músculo do cação, da extração com água, este resultado da retenção efetiva de $60,31 \%$, indicado na Tabela 2, pode também estar subestimado, por uma ação redutora parcial do Se pelo $\mathrm{NaBH}_{4}$, de acordo com Sullivan (1995). O Se ${ }^{0}$, insolúvel e estável, o $\mathrm{Se}^{2-}$ (ex.: $\mathrm{Na}_{2} \mathrm{Se}$ ), solúvel, e ainda, o hidreto volátil, poderiam ter sido formados e eliminados durante o processo de remoção do Hg (REILLY, 1995; SULLIVAN, 1995). Mesmo subavaliadas, as retenções do Se observadas são quantitativamente significativas e importantes para os propósitos nutricional inerente e de proteção em relação à toxidez do Hg.

A interação Hg-Se não é inteiramente entendida, a ponto de oferecer fundamentos concretos para uma efetiva proteção antitóxica do Se em relação ao Hg (CUVIN-ARALAR; FURNESS, 1991; GOYER, 1997). O Se é integrante da glutationa peroxidase, enzima que, junto com a catalase, a superóxido dismutase e a vitamina $\mathrm{E}$, dá proteção celular contra danos oxidativos, inclusive os provocados pelo Hg (GOYER, 1997; LALL, 1995). Possíveis mecanismos explicariam, em parte, a ação protetora do Se: (a) formação de complexos de Hg-Se inativos; (b) redistribuição do $\mathrm{Hg}$ no organismo (de órgãos mais sensíveis para outros de menor sensibilidade); (c) competição entre Hg e Se por sítios de ligação (alguns receptores localizados em tecidos); (d) conversão de compostos tóxicos de $\mathrm{Hg}$ em outros de menor toxidez (demetilação do $\mathrm{MeHg}$ no fígado); (e) diminuição do efeito inibitório do metilmercúrio sobre a atividade da glutationa peroxidase; e, (f) por prevenção de danos causados por radicais livres gerados pelo $\mathrm{Hg}$ na membrana celular (CUVIN-ARALAR; FURNESS (1991); GOYER (1997).
A complexação $\mathrm{Hg}$-Se e a redistribuição do $\mathrm{Hg}$ são considerados os mais importantes entre os mecanismos apontados anteriormente. A formação de um complexo de Hg-Se-proteína poderia explicar a relação 1:1 encontrada entre o $\mathrm{Hg}$ e $\mathrm{Se}$ em fígado de mamíferos marinhos, com decréscimo correspondente na toxicidade relativa ao Hg. Isto seria decorrente do fato de o complexo Hg-Se ligado à proteína ser muito menos tóxico que o MeHg. A absorção do Hg não é diminuída pela presença de Se, tampouco este último aumenta a eliminação do primeiro. O que há é uma redistribuição do $\mathrm{Hg}$ para tecidos menos sensíveis (ex.: músculo), conferindo algum grau de proteção (PEAKALL; BURGER, 2003).

Nenhuma correlação que fosse significativa entre o Hg e o Se muscular de peixes, crustáceos e moluscos foi encontrada (BARGHIGIANI et al.,1991; PLESSI; BERTELLI; MONZANI, 2001), que pudesse ajudar no entendimento da interação entre estes elementos. Uma maior concentração de $\mathrm{Hg}(\mathrm{p}<0,05)$ foi encontrada em peixes, comparada às verificadas em crustáceos e moluscos; por outro lado, de acordo com Plessi, Bertelli e Monzani (2001), não houve diferença ( $p>0,05)$ em relação ao Se para os três tipos de pescado, concordando com os resultados da Tabela 1.

Como na grande maioria do pescado marinho o Se muscular está estequiometricamente em excesso em relação ao $\mathrm{Hg}$, tem sido cogitado que o consumo de pescado (peixes, crustáceos e moluscos) não expõe o consumidor aos efeitos tóxicos do Hg. A elucidação da interação entre $\mathrm{Hg}$ e Se é necessária para melhor entendimento da ação protetora do Se na exposição do consumidor ao Hg (PEAKALL; BURGER, 2003; RUITER, 1995).

Particular preocupação deve ser reservada aos peixes predadores - cação, atum, swordfish -, que têm a capacidade de acumular $\mathrm{Hg}$ muscular em excessivas e perigosas quantidades e, nesta condição, são impróprios para serem usados como alimento. Visto que o $\mathrm{Hg}$ tem sido detectado em cações em quantidades $>0,5 \mu \mathrm{g} \cdot \mathrm{g}^{-1}$, em cerca de $72,5 \%$ dos casos (TENUTA-FILHO; NASCIMENTO, 2007), os níveis de Se observados nos cações analisados (Tabela 1) não estão numa relação estequiométrica suficiente para estabelecer interação com o $\mathrm{Hg}$, promovendo a proteção tóxica esperada.

\section{Conclusões}

Os níveis de Se encontrados nas espécies estudadas estão dentro da faixa de ocorrência indicada na literatura, para peixes marinhos em geral. Os níveis de Se observados nos cações não estão numa relação estequiométrica suficiente para estabelecer interação com o Hg, proporcionando a proteção tóxica esperada. A retenção efetiva do Se após extração com água, medida em cação-mangona, foi considerada apreciavelmente elevada $(68,81 \%)$.

\section{Agradecimento}

Ao Conselho Nacional de Desenvolvimento Científico e Tecnológico - $\mathrm{CNPq}$, pelo apoio financeiro. 


\section{Referências bibliográficas}

ÅKESSON, B.; SRIKUMAR, T. S. Occurrence of low-molecularweight and high-molecular-weight selenium compounds in fish. Food Chemistry, v. 51, n. 1, p. 45-49, 1994.

ASSOCIATION OF OFFICIAL ANALYTICAL CHEMISTS - AOAC. Official methods of analysis of AOAC International. 16 ed. Arlington, 1995. p. 1-23. (v. 2, cap. 39)

BARGHIGIANI, G. et al. Mercury assessment and its relation to selenium levels in edible species of the Northern Tyrrhenian Sea. Marine Pollution Bulletin, v. 22, n. 8, p. 406-409, 1991.

BROWN, K. M.; ARTHUR, J. R. Selenium, selenoproteins and human health: a review. Public Health Nutrition, v. 4, n. 2B, p. 593-599, 2001.

CAPPON, C. J. Speciation of selected trace elements in edible seafood. In: NRIAGU, J. O.; SIMMONS, M. S. (Eds.). Food contamination from environmental sources. New York: John Wiley, 1990. p. 145-195. (Advances in Environmental Science and Technology, 23).

CHICOUREL, E. L. et al. Mercúrio em pescado comercializado em São Paulo-SP, Brasil. Ciência e Tecnologia de Alimentos, v. 15, n. 2, p. 144-149, 1995.

COHEN, G. B.; SCHRIER, E. E. Removal of mercury from fish protein concentrate by sodium borohydride reduction. Journal of Agricultural and Food Chemistry, v. 23, n. 4, p. 661-665, 1975.

CUVIN-ARALAR, M. L. A.; FURNESS, R. W. Mercury and selenium interaction: a review. Ecotoxicology and Environmental Safety, v. 21, n. 3, p. 348-364, 1991.

FERREIRA, K. S. et al. Concentrações de selênio em alimentos consumidos no Brasil. Revista Panamericana de Salud Publica, v. 11, n. 3, p. 172-177, 2002.

FOLCH, J.; LESS, M.; STANLEY, S. A simple method for the isolation and purification of the total lipids from animal tissues. Journal of Biological Chemistry, v. 226, n. 1, p. 497-509, 1957.

FOSTER, L. H.; SUMAR, S. Selenium in health and disease: a review. Critical Reviews in Food Science and Nutrition, v. 37, n. 3, p. 211-228, 1997.

FUNDACENTRO. Brasil espera pescar mais de sessenta mil toneladas de sardinha em 2007. Florianópolis-SC, 2007. Disponível em: <http: br/acquaforum/principal/ver_noticias. php?not=1278>. Acesso em: 28 fev. 2007.

GOYER, R.A. Toxic and essential metal interactions. Annual Review of Nutrition, v. 17, p. 37-50, 1997.

HAARD, N. F. Composition and nutritive value of fish proteins and other nitrogen compounds. In: RUITER, A. (Ed.). Fish and fishery products: composition, nutritive properties and stability. Wallingford: CAB International, 1995. cap. 3, p. 77-115.
JOHANSSON, K.; LUO, X.; OLIN, A. Rapid one-step derivatization of $\mathrm{Se}$ (VI) to a piazselenol for the spectrofluorimetric determination of selenium in biological material. Talanta, v. 42, n. 12, p. 1979-1987, 1995.

KITAHARA, S. E. et al. Mercúrio total em pescado de água-doce. Ciência e Tecnologia de Alimentos, v. 20, n. 2, p. 267-273, 2002.

LALL, S. P. Macro and trace elements in fish and shellfish. In: RUITER, A. (Ed.). Fish and fishery products: composition, nutritive properties and stability. Wallingford: CAB International, 1995. p. 187-213. (cap. 6)

MAHER, W. A. Characteristics of selenium in marine animals. Marine Pollution Bulletin, v. 16, n. 1, p. 33-34, 1985.

MURPHY, E. W.; CRINER, P. E.; GRAY, B. C. Comparisons of methods for calculating retentions of nutrients in cooked foods. Journal of Agricultural and Food Chemistry, v. 23, n. 6, p. 1153-1157, 1975.

PEAKALL, D.; BURGER, J. Methodologies for assessing exposure to metals: speciation, bioavailability of metals, and ecological host factors. Ecotoxicology and Environmental Safety, v. 56, n. 1, p. 110-121, 2003.

PLESSI, M.; BERTELLI, D.; MONZANI, A. Mercury and selenium content in selected seafood. Journal of Food Composition and Analysis, v. 14, n. 5, p. 461-467, 2001.

RAYMAN, M. P. The importance of selenium to human health. Lancet, v. 356, n. 9225, p. 233-241, 2000.

REILLY, C. Selenium in food and health. London: Blackie Academic and Professional, 1995. 338 p.

RUITER, A. Contaminants in fish. In: Fish and fishery products: composition, nutritive properties and stability. Wallingford: CAB International, 1995. cap. 9, p. 261-285.

SULLIVAN, E. A. Hydrides. In: KIRK-OTHMER Encyclopedia of Chemical Technology. 4 ed. New York: John Wiley, 1995. v. 13, p. 606-629.

TENUTA FILHO, A.; NASCIMENTO, E. S. Mercúrio em cação: ocorrência e possibilidade de remoção. Brazilian Journal of Food Technology, v. 10, n. 2, p. 78-85, 2007.

VLIEG, P. Selenium concentration of the edible part of 74 New Zealand fish species. Journal of Food Composition and Analysis, v. 3, n. 1, p. 67-72, 1990.

WHEATON, F. W.; LAWSON, T.B . Processing aquatic food products. New York: John Wiley, 1985. 517 p.

ZHENG, O. et al. A new reagent for determining trace selenium by gas chromatography: 1,4-dibromo-2,3-diaminonaphthalene. Talanta, v. 33 , n. 5 , p. $443-444,1986$. 